\title{
Interactive comment on "The effect of seasonally and spatially varying chlorophyll on Bay of Bengal surface ocean properties and the South Asian Monsoon” by Jack Giddings et al.
}

\section{Anonymous Referee \#1}

Received and published: 19 June 2020

\section{General comments}

Giddings et al. used an atmospheric general circulation model coupled to an ocean mixed layer model to assess the impact of spatial and seasonal variability of biological radiative heating on the seasonal monsoon in the Bay of Bengal (BoB). Two experiments were performed, one in which the attenuation depth of visible radiation is constant, the other in which the attenuation for visible radiation is spatially and seasonally varying based on a monthly climatology of satellite chlorophyll measurements. The authors find that imposing spatially- and seasonally-varying attenuation depths gives rise to modified patterns of sea surface temperature (SST) and moisture flux, which at 
some location contribute to improving the precipitation biases of the model. By means of a mixed layer heat budget analysis, the authors find that the depth of visible light attenuation relative to that of the mixed layer is a crucial factor in determining whether the radiative heating will significantly affect the SST.

I find that the manuscript is promising in its methodological concept and that it could make an interesting contribution to the research field addressed by WCD. The ideas, tools and concepts are not fully novel, but their application to the South Asian monsoon has not yet been extensively elucidated in past research. However, I find that the manuscript should be improved in terms of clarity, and that the results are not conveyed in a satisfying manner.

\section{Specific comments}

In order to support the conclusions, I believe that five aspects should be improved:

1) The authors should state more clearly the aims and the key results of the study as well as their contribution to the research field. It is not clear to me whether the focus is on improving model biases or on the mechanistic understanding of how biological radiative heating affects the monsoon in the BoB.

2) In the Introduction, the physical mechanisms driving the mean seasonality of the South Asian monsoon should be better explained. Fig. 1 could be extended to contain two panels (e.g. for summer and autumn) showing schematically the prevalent winds and ocean currents associated with the monsoon. The areas of the chlorophyll peaks could be highlighted with e.g. a box.

3) I believe the figures could be adapted to make the relevant results stand out more clearly. In particular, I find Fig. 4 and Fig. 8 particularly trying. The plots are extremely small (zooming on the BoB region would certainly help) and the areas, where changes are statistically significant, are difficult to see (also because of the dots used for the political boundaries, are these needed?). In addition, the large number of figures makes 
it difficult to extract the relevant information. I suggest that the authors think carefully whether all the figure panels are really necessary and whether there could be a more effective way of showing the information. An idea could be to show seasonal cycles of these variables spatially-averaged over dynamically-consistent regions (e.g. Irrawaddy Delta region/SMC region, or open ocean/coastal, or north/south) and to show the difference maps only for selected months, seasons or for a subset of variables. Having the whole seasonal cycle on one plot (as in Fig. 9) would make it easier to relate the response of the physical variables to the modified attenuation depth.

4) In describing the results, the authors should make an effort to sift through the plentiful information provided by the model simulations in order to highlight the relevant messages. Concluding each of the Results' subsections with a few summarizing sentences would help, as well as including a final schematic showing the critical mechanisms emerging from this study.

5) If I understand correctly, a key take home message of the paper is that the interplay between the biologically-driven heating and the depth of the mixed layer, as well as the timing of the biological heating with respect to the seasonality of the monsoon, determine whether phytoplankton will exert a strong or weak influence on the monsoon. In this respect, different regions behave differently because of their underlying stratification and phytoplankton seasonality. This is an intriguing point which I think the authors should expand and discuss more.

From a methodological point of view, I find it somewhat disturbing that the authors define their model as a "coupled ocean-atmosphere general circulation model (GCM)" since the ocean model is not a GCM but an ocean mixed layer configuration containing no advective processes. The opportunities and disadvantages of this methodology should be clearly expressed.

Line-by-line comments:

Lines 15-24: please state the aims of the study more clearly. Also, a few more words 
could be said on the length of the simulations. As mentioned earlier, defining the model as a "coupled ocean-atmosphere model" is misleading, since it brings to the mind a WCDD coupled ocean-atmosphere GCM. I suggest being more specific.

Introduction (lines 28-104): I think the Introduction needs more structure. The fact that the paper deals with the effects of biological radiative heating on the monsoon comes quite late (line 84). My suggestion is to have a first overarching paragraph on the relevance of the monsoon and on overlooked feedbacks between biology and the monsoon. The paragraphs afterwards can go into more detail on the physical and biological properties of the region (and here, please enrich your text by referring to an updated Fig. 1 - see my point 2 of the specific comments). I also think that the contribution you want to make to the research field should be better highlighted in the last paragraph.

Lines 101-102: I believe Wetzel et al. (2006) used a coupled GCM, whereas your study actually does not. This statement is therefore incorrect.

Lines 127-128: I suggest adding a more expanded definition of h1 and h2, for instance: the e-folding depth, or attenuation depth, is the depth where surface radiation decreases by $1 / \mathrm{e}$ (or $37 \%$ ) of its initial surface value.

Lines 138-139: Please state already here which Jerlov water type is used in the simulation with constant attenuation depth.

Lines 141-145: How does the relaxation of ocean temperature to observations affect your results? Which is the relaxation time scale and how sensitive are your results to the relaxation time scale?

Lines 155 and later line 181: why say "h2 values (i.e. chlorophyll concentration)"? It is clearly not the same quantity.

Line 173: Fig. 1 could show in gray shading the grid points in which chl-a was not determined by satellite.

Interactive

comment

Printer-friendly version

Discussion paper 
Lines 184-185: 30-years of simulation sounds quite short, also considering that computational costs are not very high (line 149). Is the model in an adjusted state?

Lines 200-212: I think these two paragraphs should go in Section 2.2. Indeed, at the first sentence of Section 2.2 I was already wondering how you compute h2 based on chlorophyll data.

Lines 213-226: This section doesn't read well, as it contains three very different sets of information (statistical significance computation, VIMF computation, regridding of observed precipitation). Please restructure. Part of the information could maybe go in the figure captions.

Line 245: Unless I'm mistaken, Fig. 6 is cited before Fig. 5.

Lines 251-252: please explain the mechanism better: despite the SST increase, evaporation decreases because air humidity increases?

Line 255 and 265: "caused by" instead of "corresponds"?

Lines 255-259 (same for 289-291): I suggest not mixing mechanism understanding and bias improvement in one paragraph. Indeed, maybe all the part on bias improvement could be shifted to the discussion?

Lines 263-272: This paragraph is somewhat cumbersome. I suggest condensing the relevant information in fewer sentences.

Line 274: co-located?

Line 274: no comma after whereas?

Line 275: Increased SST also speeds up evaporation.

Lines 300-306: This part seems more suitable for the Discussion section.

Section 3.3 (lines 309-371): if I understand correctly, Eq. 4 is used to estimate the relative contribution of attenuation depth, incoming shortwave radiation and MLD changes

Interactive

comment

\section{C5}

Printer-friendly version

Discussion paper 
on the radiative heating. Throughout the section, you then refer to these results only in the text. I think it would be more useful to see the relative contribution of these processes in a separate figure. For instance, in Fig. 11 you could show, instead of the Qsw and MLD changes, the relative contribution that Qsw and MLD have on the RHR.

Line 320: I'm acquainted with $\mathrm{cp}=3850 \mathrm{~J} /\left(\mathrm{kg}^{*} \mathrm{~K}\right)$. Please clarify.

Interactive

Line 324: "in" too much

comment

Lines 329-330: please be more explicit on why you selected these two regions.

Lines 356-370: please state more explicitly why a deeper MLD leads to smaller biologically-driven changes in SST. If I understand correctly, the mechanism you are referring to is: an increased radiative heating in the upper levels caused by biology leads to radiative cooling in the layers below. A MLD deeper than the attenuation depth causes this dipole to mix, and therefore (assuming constant Qsw) the net effect of biological heating on ML temperature should be null. To that one should also add the fact that higher surface SSTs lead to increased ocean heat losses by evaporation. Therefore, on annual average the areas of high biology (and deep MLD) should actually experience surface cooling. I wonder whether you see this effect in your model?

Lines 356-371: It is intriguing to see that the presence of biology and associated radiative heating do not modify the MLD. Could you comment on that?

Discussion-Conclusions: The authors could consider joining the Discussion and the Conclusions. My suggestion: first start with a paragraph summarizing the main results (as in lines 435-455), then continue with the discussion points, and finally conclude with the open questions and outlook. As it is now, some parts of the Conclusions seem more apt to be in the Discussion.

Printer-friendly version

Lines 388-389: The MLD changes of $1 \mathrm{~m}$ cannot readily be seen in Fig. 11. It would be more useful to show spatially averaged MLD anomalies as suggested in point 3 of my specific comments. 
Lines 390-391: This is an interesting statement, which would imply that in coastal regions the local effect of high chlorophyll dominates the perturbation minus control WCDD anomalies in stratification, whereas in the open ocean remote effects through wind speed changes play a leading role. Some more analysis should be shown to substantiate this statement.

Lines 423-425: The discussion on the effect of subseasonal chlorophyll variability on the Boreal Summer Intraseasonal Oscillation comes somewhat out of the blue, since you have not mentioned any form of intraseasonal or interannual variability in the paper. Please introduce this paragraph better to put the reader in the picture.

Lines 430-431: Which feedbacks and processes are you missing by using an ocean mixed layer model instead of an ocean GCM (and by using an imposed chlorophyll distribution instead of an interactive biogeochemical model)? Do you expect the results from your modeling setting to be rather overestimating or underestimating the "true" response?

Line 436: Please be more specific on the model you used. (Coupled oceanatmosphere GCM is misleading).

Lines 450-454: The inclusion of biological heating reduces the precipitation biases in some locations, which is good, but does it increase the biases elsewhere? (e.g. over Myanmar?)

Interactive comment on Weather Clim. Dynam. Discuss., https://doi.org/10.5194/wcd-2020-15, 2020. 